RESEARCH ARTICLE

\title{
Fractional Anisotropy Brain Changes in Spasticity after Peripheral Injections of Botulin
}

\author{
Miguel Angel Bertoni ${ }^{1^{*}}$, Mohammed Sakel ${ }^{2}$ and Ali Hojjatoleslami ${ }^{3}$ \\ ${ }^{1}$ Department of Radiology, East Kent Hospitals University NHS Foundation Trust, University of Kent, UK \\ ${ }^{2}$ Department of Neurorehabilitation, East Kent Hospitals University NHS Foundation Trust, UK \\ ${ }^{3}$ Medical Imaging, University of Kent, UK
}

*Corresponding author: Miguel Angel Bertoni, Department of Radiology, East Kent Hospitals University NHS Foundation Trust, University of Kent, UK

\begin{abstract}
Purpose: To assess central fractional anisotropy changes of the white matter in subjects with spasticity treated with botulin toxin.

Materials and Methods: Seven subjects with spasticity that developed 2-22 years after cerebrovascular accidents (CVA) were studied with 3T MRI of the brain including diffusion tractography (DTI) and fractional anisotropy (FA) measurements of the white matter of the corticospinal tracts (CST), corpus callosum (CC) and cerebral lobes of the affected and contralateral sides prior and after the injection of botulin toxin at the contours of peripheral nerves of the affected side.
\end{abstract}

Results: All 7 subjects improved their preexisting spasticity after the injections of botulin toxin. 4 out of 7 showed FA changes post injection in the CST of the affected side. The latter had CVAs occurring 5-15 years prior to the development of spasticity. 3 out of 7 subjects did not show significant FA changes post-treatment. They had CVAs occurring less than 5 years and more than 15 years prior to the development of spasticity. There was regression between 'years of evolution', as a dependent variable, and the square of pre and post injection differences of FA on the contralateral CST, as well as white matter of the parietal and frontal lobes as independent variables.

Conclusion: Botulin toxin injections improved spasticity in all the subjects of our cohort. There also were FA changes in the white matter of the CST of the affected side in those subjects with CVAs happened between 5-15 years prior to the development of spasticity, while those with lesions of less than 5 years and more than 15 years did not show statistically significant FA changes post treatment.

\section{Keywords}

Fractional anisotropy, Spasticity, Botulin, Diffusion tractography, Magnetic resonance imaging

\section{Introduction}

Spasticity is a physiological consequence of damage to nerve cells or their pathways that control muscle movement within the brain or spinal cord. It results in uncontrollable stiffening or straightening of muscles, shock-like contractions of all or part of a group of muscles and abnormal muscle tone which can have harmful effects such as pain, deformity, impaired function and severe disability with costly consequences. In severe cases it requires perineural injections of botulin toxin to alleviate disability and to improve quality of life.

Although the signs and symptoms caused by spasticity that affect activities of daily living are well recognized, the understanding of its causes and when it develops with respect to the time of onset of the neurological lesion are less well understood [1].

It is estimated that around $75 \%$ of patients with severe traumatic brain injury, $20 \%$ of stroke survivors and $60 \%$ of subjects with moderate to severe Multiple Sclerosis require specific spasticity treatment [2].

The neurological lesions of subjects with spasticity can be studied with non-invasive imaging techniques, particularly those related to MRI. Diffusion tractography imaging (DTI) is an advanced magnetic resonance imaging (MRI) tool based on the diffusion of water molecules within cells. In the case of neurons, it helps to image nerve bundles of white matter tracts [3-8]. Furthermore, it also provides quantitative markers

Citation: Bertoni MA, Sakel M, Hojjatoleslami A (2018) Fractional Anisotropy Brain Changes in Spasticity after Peripheral Injections of Botulin. Int J Physiatry 4:015. doi.org/10.23937/25724215.1510015

Accepted: November 20, 2018: Published: November 22, 2018

Copyright: (c) 2018 Bertoni MA, et al. This is an open-access article distributed under the terms of the Creative Commons Attribution License, which permits unrestricted use, distribution, and reproduction in any medium, provided the original author and source are credited. 
such as fractional anisotropy (FA) and Anisotropy diffusion coefficient or Apparent diffusion coefficient (ADC) [9]. In the cerebral white matter, contributions to these indicators come from fibre arrangements, degree of myelination, and axonal integrity [10], and are useful indicators to assess the severity of already known pathological conditions [11-13]. As an indicator of myelin sheath integrity, FA has already been correlated with electrophysiology studies, confirming the technique as particularly sensitive to distal motor latency and sensory nerve conduction velocity changes [14]. FA has been also used as a reliable indicator to evaluate the normalization of neuron structure in white matter lesions after therapy in demyelinating conditions showing neuroprotective effects of some drugs [15], and to assess pathological changes reflecting alteration of neuron integrity in neurodegenerative diseases [16] and traumatic brain injury [17].

ADC measurements for complicated systems as living tissues depend on the diffusion time and weighting used, or b factor, in a given volume unit or voxel. In this study, the diffusion tensor expressed by FA was preferred over $A D C$ as the former reflects the underlying directional diffusion properties of the tissue independently of its orientation in the magnetic field while Te latter is influenced by how the subject has been placed in the scanner magnet and gradient coils $[18,19]$.

\section{Purpose}

The main purpose of this pilot study was to evaluate if there were quantitative changes in the nerve bundles of the central white matter in subjects who developed spasticity after a CVA which could be identified with DTIFA after the injection of botulin toxin on the affected side.

A secondary purpose of this work was to investigate if there was a relationship between years of evolution after the lesion and FA values in the central white matter.

\section{Materials and Methods}

The Bioethics approval for this study was obtained from the Health Research Authority, NRES Committee South Central-Oxford, in the United Kingdom (13/ SC/0561; IRAS 140861). The institutional approval was granted by Research \& Development of East Kent Hospitals University NHS Foundation Trust (2013/ RADIO/02) and individual subject consents were obtained before the first scan in what concerns imaging aspects of this pilot study. Separate individual consents were obtained by the Department of Neurorehabilitation before the treatment.

The initial cohort included 12 subjects who had developed spasticity due to upper motor neuron lesions. They had single hemispheric post ischaemic or haemorrhagic parenchymal sequelae affecting the gangliocapsular area with extension to the cerebral lobes. The lesions took place within a range from 1 to 25 years before development of a handicapping degree of spasticity requiring peripheral injections of botulin toxin. The subjects were studied with Magnetic Resonance Imaging of the head before and after the treatment.

The spasticity scores were obtained according the Disability Assessment Scale [20] pre and post-injection.

Two subjects were excluded immediately after the initial MRI series for technical reasons (metallic fragments causing inhomogeneity in the magnetic field). The remaining ten were studied with MRI of the brain at a magnetic field strength of 3 Tesla and following a protocol that included localizers, isovolumetric T1, isovolumetric T2, axial and coronal FLAIR, axial Proton Density and T2, axial susceptibility weighted images (SWI) and diffusion tractography series (DTI) with 32 directions (Table 1 ), before and eight weeks after the peripheral perineural injection of botulin toxin $(200$ units, diluted in $4 \mathrm{ml}$ of saline solution $9 \%$ ).

Three subjects were excluded after the full initial scan due to poor imaging quality (movement artifacts or inability to complete the imaging protocol). Seven subjects were included and were studied before and after the injections. The obtained images were post processed in an independent workstation using Syngo

Table 1: MR imaging protocol.

\begin{tabular}{|c|c|c|c|c|c|}
\hline \multirow{2}{*}{\begin{tabular}{|l|} 
MR unit \\
Series \\
\end{tabular}} & \multicolumn{5}{|c|}{ Siemens ${ }^{\circledR}$ skyra ${ }^{\circledR}$ with a magnetic field strength of 3 tesla } \\
\hline & TR & TE & FoV & NEX & Resolution \\
\hline Localizers & \multicolumn{5}{|c|}{ Gradient echo T1, large FOV, quick acquisition time in all three planes as per vendor } \\
\hline Axial T2 (tse) & 5000 & 88 & $22 \mathrm{~cm}$ & 1 & $0.4 \times 0.4 \times 5 \mathrm{~mm}$ \\
\hline Axial T1 (tse) & 400 & 7.1 & $22 \mathrm{~cm}$ & 2 & $0.6 \times 0.6 \times 5 \mathrm{~mm}$ \\
\hline Axial FLAIR & 9000 & 81 & $22 \mathrm{~cm}$ & 2 & $0.7 \times 0.7 \times 5 \mathrm{~mm}$ \\
\hline Coronal FLAIR (mpRAGE) & 4680 & 84 & $22 \mathrm{~cm}$ & 1 & $0.5 \times 0.5 \times 5 \mathrm{~mm}$ \\
\hline Axial 3D T1 (SPACE) & 1900 & 2.49 & $22 \mathrm{~cm}$ & 1 & $0.9 \times 0.9 \times 0.9 \mathrm{~mm}$ \\
\hline Axial 3D T2 & 3200 & 412 & $22 \mathrm{~cm}$ & 1 & $1 \times 1 \times 1 \mathrm{~mm}$ \\
\hline Axial SWI & 400 & 4.92 & $22 \mathrm{~cm}$ & 1 & $3 \times 3 \times 3 \mathrm{~mm}$ \\
\hline Axial DWI 32 directions & 700 & 95 & $22 \mathrm{~cm}$ & 1 & $1.7 \times 1.7 \times 4 \mathrm{~mm}$ \\
\hline
\end{tabular}

FLAIR: Fluid Attenuation Inversion Recovery; SWI: Susceptibility Weighted Images; DWI: Diffusion Weighted Imaging; TR: Time of Repetition; TE: Time of Echo; FoV: Field of View; NEX: Number of Experiments.

The acronyms between brackets indicate the name of the pulse sequences as per the software of the magnetic resonance unit used for the study (Siemens $\left.{ }^{\circledR}\right)$. 
$\mathrm{Via}^{\circledR}$ software to image the corticospinal tracts of the affected and contralateral sides (Figure 1 and Figure 2 ), the corpus callosum (Figure 3) and the tracts in the white matter of the frontal, parietal, temporal and occipital lobes of the affected and contralateral sides. The seeding points for tractography [21] were placed in the rostral cerebral peduncles on axial plane for the corticospinal tracts, in the corpus callosum on the sagittal plane, and in the white matter of the cerebral lobes within the boundaries set by the Talairach Atlas on axial plane. Fractional anisotropy values were obtained for each of the tracts. Each measurement was repeated three times to ensure consistency.

The tabled variables included age, gender, spasticity score, presence of Wallerian degeneration, score of disability assessment scale pre and post injection and fractional anisotropy (FA) values of the corticospinal tracts (CST), corpus callosum (CC), frontal (FL), parietal

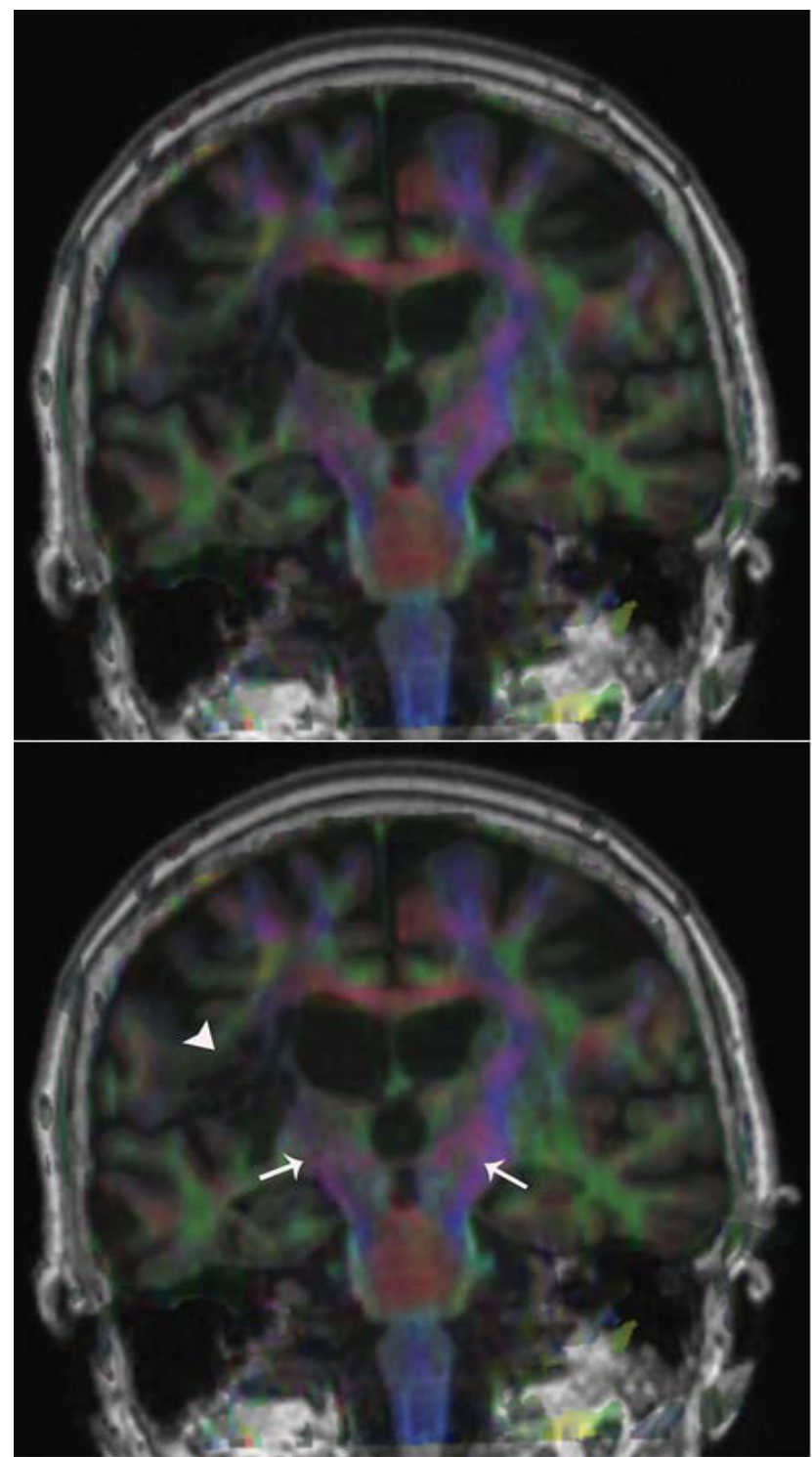

Figure 1: FA maps fused to coronal T1 sectioning rostrally the cerebral peduncles showing CSTs in blue (white arrows). Note the lesion (white arrowhead) on the affected side on the patient's right (left side of the image) and how it extends to the right CST.
$(P L)$, temporal $(T L)$ and occipital lobes (OL) of the affected (aff) and contralateral (cont) side. In the case the $\mathrm{CC}$, as a midline structure, there were single pre and post injection measurements.

The square powers of differences between pre and post injection $F A$ values of each measurement were also tabled.

Calculations carried out were basic statistics, potency tests for dependent samples, T-tests for dependent variables and Forward Stepwise Multiple Regression analysis. The latter considered the years elapsed from the onset of the lesion to the development of spasticity as a dependent variable and the transformed variables as independent, for each one of the areas, which included CST, CC, FL, PL, TL and OL of the affected and contralateral sides. All the quantitative variables were plotted in graphs jointly and separately on the $y$-axis versus years of evolution on the $x$-axis. The fittings were both linear and least squares in all the graphs. After obtaining the results as shown below, all the FA values were entered multiplied by $1000(x=F A \times 1000)$ to do the calculations with integer numbers.

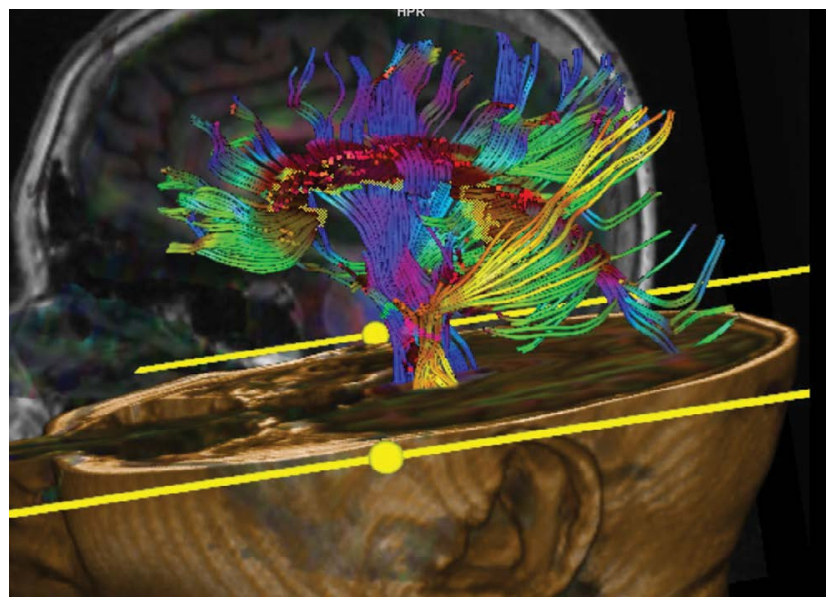

Figure 2: DTI fused to 3D T1 showing CSTs (yellow and blue) and CC (purple).

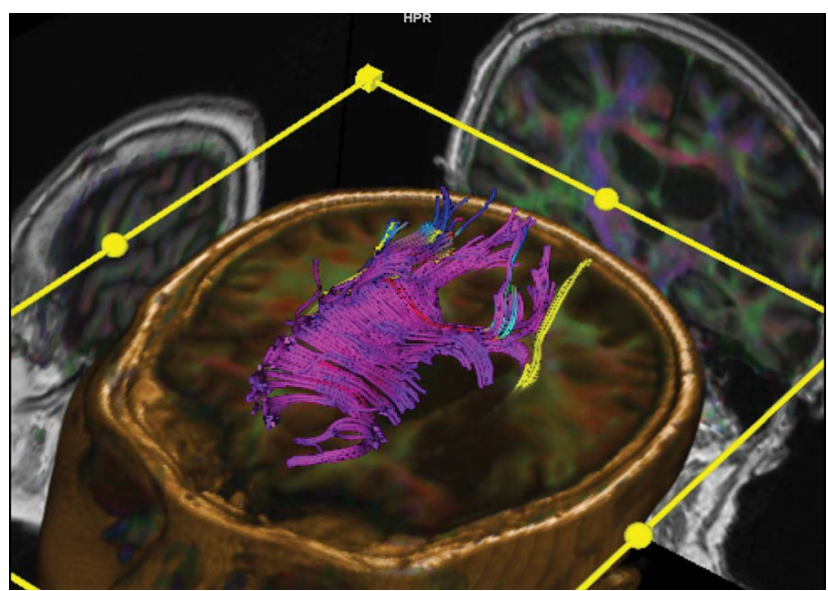

Figure 3: DTI fused to 3D T1 showing CC in purple. Some of its fibres are affected by the lesion on the right side of the patient (and also right side of the photo). 


\section{Results}

The seven subjects in this cohort included 2 males and 5 females, with an average age of 49 years (24-71) and an interval evolution of 10 years (1-25) since the onset of the causative central nervous system lesion. Two had imaging signs of Wallerian degeneration with bright signal on T2 and FLAIR in the CST at the rostral aspect of the cerebral peduncles on the affected side (Table 2).

The degree of spasticity improved in all subjects after the injection of botulin toxin. Their mean spasticity score in the Disability Assessment Scale improved from 2.5 (23 ) previously to the injection to 1 (0-2) post-injection.

Pre-injection mean FA values for CC were 0.448 and on the affected side 0.462 for CST, 0.355 for FL, 0.372 for PL, 0.363 for PL, and 0.388 for OL. Pre-injection FA values on the contralateral side were 0.485 for CST, 0.370 for $\mathrm{FL}, 0.406$ for PL, 0.413 for TL and 0.395 for OL. Post injection FA for CC was 0.457. On the affected side FA was 0.470 for CST, 0.340 for FL, 0.358 for PL,

Table 2: Qualitative variables in the cohort.

\begin{tabular}{|l|l|l|}
\hline Gender & Males & Females \\
\hline Wallerian degeneration & 2 & 5 \\
\hline & Yes & No \\
\hline 2 & 5 \\
\hline
\end{tabular}

0.380 for TL and 0.394 for OL. Post-injection FA values for the contralateral side were 0.480 for CST, 0.388 for FL, 0.411 for PL, 0.408 for TL and 0.420 for OL (Table 3).

The mean values of transformed variables were 833 for CC and on the affected side 203 for CST, 972 for FL, 783 for PL, 6302 for TL and 1143 for OL. The mean values of post injection transformed variables on the contralateral side were 647 for CST, 984 for FL, 1112 for PL, 176 for TL and 1954 for OL (Table 4). On (Figure 4) the linear graph shows FA values of affected CST tracts on $y$-axis versus years of evolution on the $x$-axis before and after the injection of botulin toxin, with least squares fitting. On (Figure 5) the box graph shows the distribution of FA values of affected CSTs pre and post injection for all 7 subjects.

The obtained power calculation of two-means $T$ test for dependent samples was 0.81 with an alpha error of 0.05 .

$T$ tests for dependent variables, considering the pre and post injection spasticity scores (DAS), were significant $(t=7.07)$. $T$ tests for dependent variables were not significant when all seven subjects were considered (Table 5), although they became significant when only the subjects with a lesion evolution between 5-15 years were included (4 out of 7 subjects) (Table $6)$; the subjects with lesions of less than 5 years and

Table 3: Quantitative variables in the cohort.

\begin{tabular}{|c|c|c|c|c|c|}
\hline \multirow[t]{3}{*}{ Variable } & \multicolumn{5}{|c|}{ Descriptive statistics for quantitative variables } \\
\hline & \multicolumn{5}{|c|}{ (FA values are converted into an integer by multiplying them by 1000 ) } \\
\hline & Valid $\mathbf{N}$ & Mean & Minimum & Maximum & Std.Dev. \\
\hline DASpre & 7 & 2.4286 & 2.0000 & 3.0000 & 0.53452 \\
\hline DASpost & 7 & 1.0014 & 0.0100 & 2.0000 & 0.57447 \\
\hline Wallerian deg & 7 & 103.5714 & 103.0000 & 104.0000 & 0.53452 \\
\hline CSTaff & 7 & 462.0000 & 424.0000 & 509.0000 & 29.30870 \\
\hline CSTcont & 7 & 485.8571 & 428.0000 & 546.0000 & 39.80548 \\
\hline CCallosum & 7 & 448.1429 & 389.0000 & 489.0000 & 38.36417 \\
\hline FLaff & 7 & 355.4286 & 308.0000 & 423.0000 & 44.35034 \\
\hline FLcont & 7 & 370.5714 & 317.0000 & 457.0000 & 52.54477 \\
\hline PLaff & 7 & 372.8571 & 304.0000 & 436.0000 & 52.11663 \\
\hline PLcont & 7 & 406.4286 & 359.0000 & 444.0000 & 32.91331 \\
\hline TLaff & 7 & 363.4286 & 283.0000 & 439.0000 & 62.15534 \\
\hline TLcont & 7 & 413.0000 & 389.0000 & 431.0000 & 17.06849 \\
\hline OLaff & 7 & 388.5714 & 339.0000 & 444.0000 & 44.01839 \\
\hline OLcont & 7 & 395.7143 & 334.0000 & 439.0000 & 40.52042 \\
\hline CST2aff & 7 & 470.1429 & 441.0000 & 501.0000 & 21.92737 \\
\hline CST2cont & 7 & 480.2857 & 458.0000 & 512.0000 & 22.17141 \\
\hline CCallosum2 & 7 & 457.0000 & 411.0000 & 486.0000 & 23.57965 \\
\hline FL2aff & 7 & 340.5714 & 245.0000 & 419.0000 & 58.86951 \\
\hline FL2cont & 7 & 388.0000 & 323.0000 & 433.0000 & 41.34408 \\
\hline PL2aff & 7 & 358.7143 & 245.0000 & 423.0000 & 68.34402 \\
\hline PL2cont & 7 & 411.5714 & 344.0000 & 442.0000 & 38.11324 \\
\hline TL2aff & 7 & 380.1429 & 308.0000 & 463.0000 & 58.57311 \\
\hline TL2cont & 7 & 408.7143 & 370.0000 & 443.0000 & 23.98412 \\
\hline OL2aff & 7 & 394.4286 & 341.0000 & 455.0000 & 37.66013 \\
\hline OL2cont & 7 & 420.5714 & 349.0000 & 469.0000 & 39.83657 \\
\hline Years & 7 & 10.5714 & 1.0000 & 25.0000 & 7.93425 \\
\hline
\end{tabular}


Table 4: Transformed variables.

\begin{tabular}{|c|c|c|c|c|c|}
\hline \multirow[t]{3}{*}{ Variable } & \multicolumn{5}{|c|}{$\begin{array}{l}\text { Descriptive statistics of transformed variables } \\
\text { (square power of pre- \& post injections FA differences) }\end{array}$} \\
\hline & \multicolumn{5}{|c|}{ [FA values are converted into an integer by multiplying them by 1000 ] } \\
\hline & Valid $\mathbf{N}$ & Mean & Minimum & Maximum & Std.Dev. \\
\hline dCSTaff & 7 & 203.571 & 1.0000 & 484.00 & 200.637 \\
\hline dCSTcont & 7 & 647.000 & 36.0000 & 1600.00 & 607.514 \\
\hline $\mathrm{dCC}$ & 7 & 833.143 & 1.0000 & 3481.00 & 1212.439 \\
\hline dFLaff & 7 & 972.571 & 4.0000 & 4761.00 & 1711.392 \\
\hline dFLcont & 7 & 984.571 & 36.0000 & 4624.00 & 1637.711 \\
\hline dPLaff & 7 & 783.000 & 1.0000 & 4096.00 & 1519.483 \\
\hline dPLcont & 7 & 1112.286 & 64.0000 & 4225.00 & 1524.096 \\
\hline dTLaff & 7 & 6302.143 & 1.0000 & 17161.00 & 7017.994 \\
\hline dTLcont & 7 & 176.000 & 9.0000 & 576.00 & 197.101 \\
\hline dOLaff & 7 & 1143.286 & 1.0000 & 3136.00 & 1181.290 \\
\hline dOLcont & 7 & 1954.857 & 9.0000 & 10816.00 & 3957.964 \\
\hline
\end{tabular}

Table 5: T tests for dependent samples in all 7 subjects, time range 1-25 years since from the causing lesion.

T-test for Dependent Samples. Time range 1-25 years

Variable Marked differences are significant at $p<0.05000$ (FA values are converted into an integer by multiplying them by 1000 )

(FA values are converted into an integer by multiplying them by 1000)

\begin{tabular}{|l|l|l|l|l|l|l|l|l|}
\hline & Mean & Std.Dv. & N & Diff. & Std.Dv.Diff. & t & df & p \\
\hline CSTaff & 462.0000 & 29.30870 & & & & & & \\
\hline CST2aff & 470.1429 & 21.92737 & 7 & -8.1429 & 12.65476 & -1.70244 & 6 & 0.139569 \\
\hline
\end{tabular}

Table 6: $T$ tests for dependent samples in 4 subjects with in a time range of 5-15 years from the causing lesion.

\begin{tabular}{|c|c|c|c|c|c|c|c|c|}
\hline \multirow[t]{2}{*}{ Variable } & \multicolumn{8}{|c|}{$\begin{array}{l}\text { T-test for dependent samples: Time range } 5-15 \text { years } \\
\text { Marked differences are significant at } p<0.05000 \\
\text { (FA values are converted into an integer by multiplying them by 1000) }\end{array}$} \\
\hline & Mean & Std.Dv. & $\mathbf{N}$ & Diff. & Std.Dv.Diff. & $\mathbf{t}$ & df & $\mathbf{p}$ \\
\hline CSTaff & 449.0000 & 27.39830 & & & & & & \\
\hline CST2aff & 466.7500 & 24.06069 & 4 & -17.7500 & 4.99166 & -7.11186 & 3 & 0.005721 \\
\hline
\end{tabular}

\section{Time of evolution vs FA of affected CSTs before and after botulin Time range $1-25$ years, 7 subjects (y axis: FA $x$ 1000)}

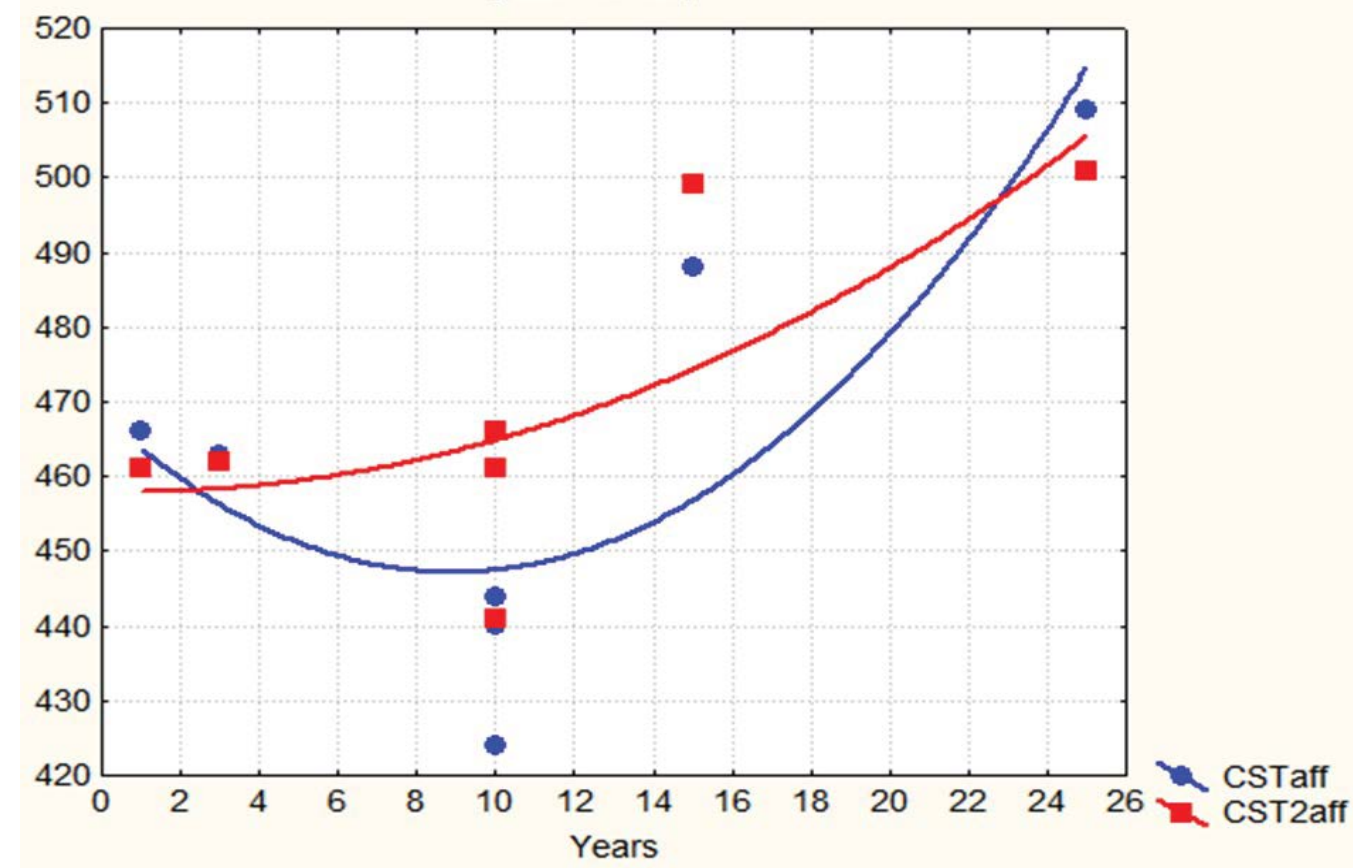

Figure 4: Time of evolution with range 1-25 years on $x$-axis vs. fractional anisotropy of the affected corticospinal tract before and after the injection of botulin in all 7 subjects. FA has been multiplied by 1000 to convert it into an integer. 
more than 15 years of evolution since onset ( 3 out of 7) were excluded (Figure 6). The latter were carried out after noting maximal FA differences in subjects within that range on graphs that plotted years of evolution on $x$-axis and pre and post-injection FA values in all four of them on $y$-axis, while no such changes were depicted for subjects with lesions that happened less than 5 and more than 15 years from the development of spasticity that affects activities of daily living (Figure 4, Figure 5, Figure 6, Figure 7 and Figure 8). Potency tests for dependent samples were again calculated including only the 4 subjects with lesions with an evolution within

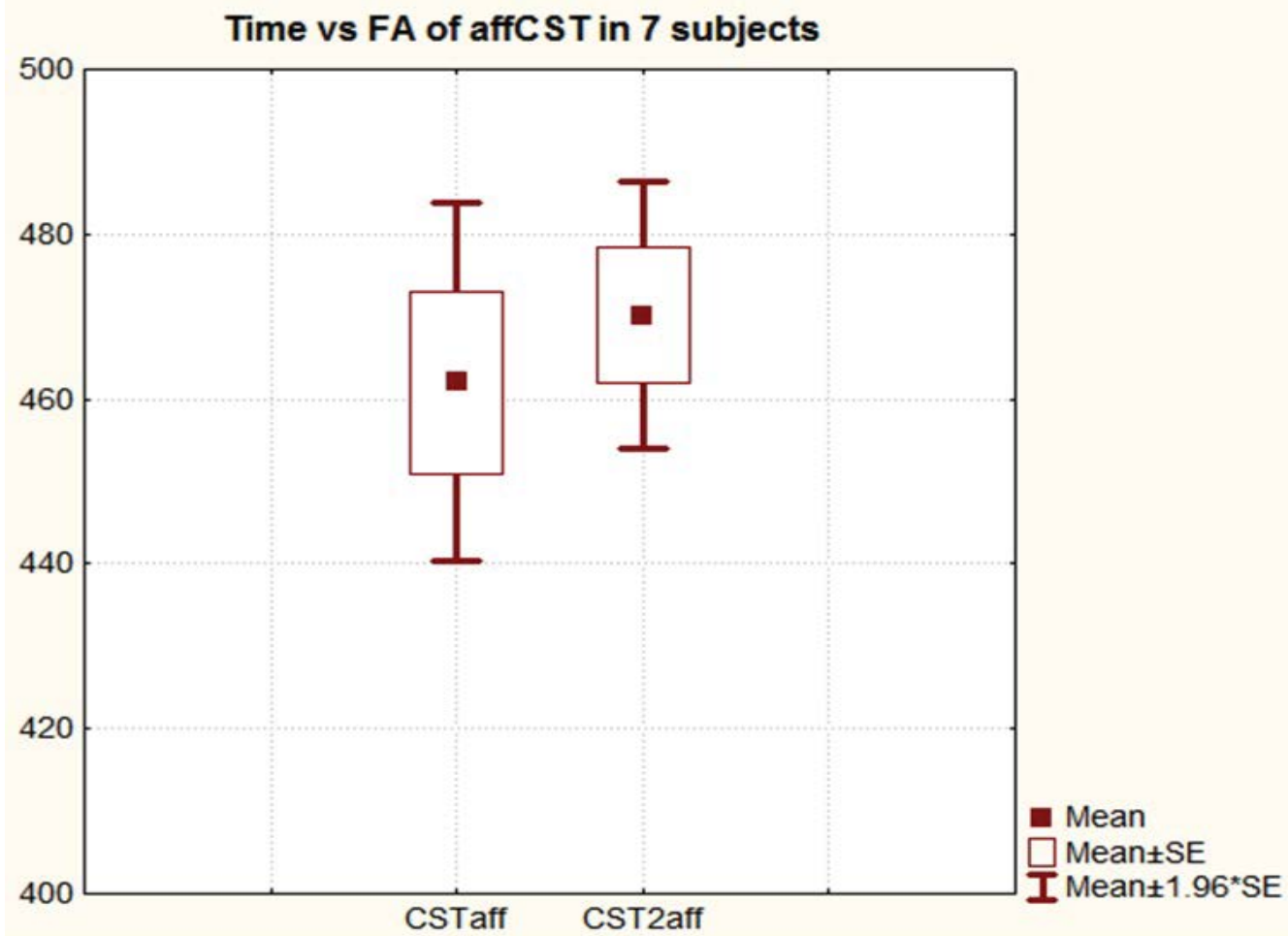

Figure 5: Fractional anisotropy of the affected corticospinal tracts pre and post botulin injection. FA is multiplied by 1000 to convert it into an integer. All 7 subjects were included. Evolution time range 1-25 years.

Time vs FA of aff CST in 4 subjects

x: 5 - 15 years; $y$ : FAx 1000

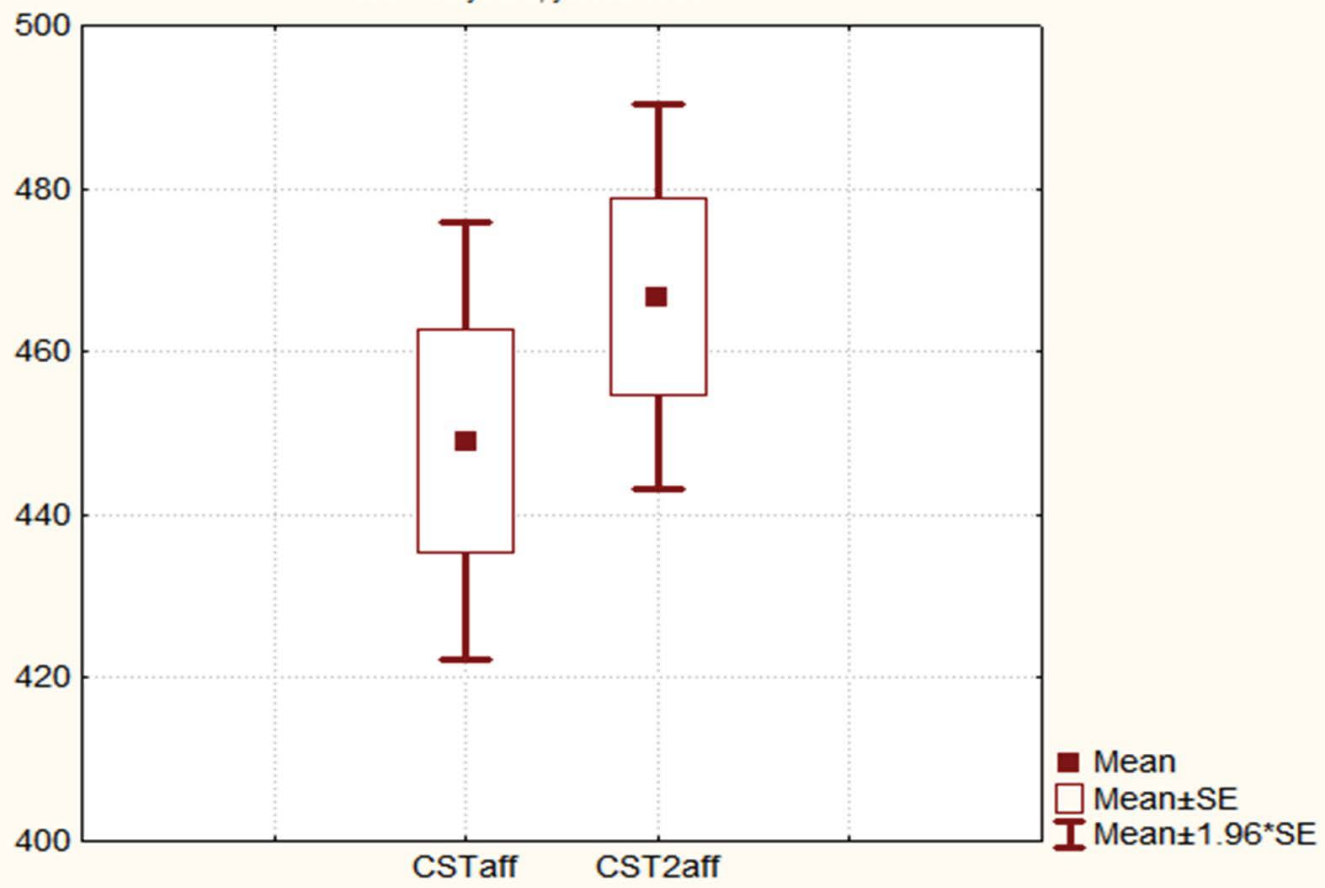

Figure 6: Fractional anisotropy values of the affected corticospinal tracts in 4 subjects, whose time of evolution after the causing lesion ranged 5-15 years. FA is multiplied by 1000 to convert it into an integer. 
a range of 5-15 years, showing similar results to those obtained when considering all 7 subjects.

Multiple regression analyses for 'years' as a dependent variable, and transformed FA differences as independent variables showed the following values: $R=$ $0.92, R^{2}=0.84$ and ${ }_{\text {adj }} R^{2}=0.69$ with $F_{(3,3)}=5.5, p<0.05$ (Table 6).

\section{Discussion}

Spasticity indicates upper motor neuron dysfunction and when it is severe it usually leads to handicapping motion restriction and serious disability [22]. It is a common problem in patients following head trauma and cerebral vascular accident (CVA) $[1,23]$ and may develop shortly after the onset of the initial lesions or several years afterwards.

Depending on its severity, spasticity may be improved by the perineural injection of botulin toxin close to the peripheral nerves controlling the affected muscle groups, mostly to improve quality of life [24] as performed with all the subjects included in this study. Post botulin injection changes may last for a variable period of time [25-27].

For these cases of subjects with handicapping spasticity, the status of white matter tracts were assessed with DTI to obtain not only qualitative assessment of the affected white matter but also a to measure FA as a quantitative indicator of the bundles microstructure $[9,10,28]$. Changes in this indicator usually predict evolution in acquired brain injuries and neuroinflammatory conditions $[4,28,29]$.
Some authors have reported central changes after peripheral treatments with botulin toxin [30-37]. However, it is unclear why FA changes post injection were identified in the four subjects of our cohort with an evolution that ranged from 5 to 15 years since the initial lesion while the remaining three, with an evolution of less than five years and more than fifteen years, did not show significant changes [38-41]. It has been shown that FA changes occur in given periods of time in linear studies of subjects with Parkinson's disease [42] and hypertonia [43]. It may be hypothesized that microstructural changes related to neuroplasticity are favoured once all the inflammatory changes after the initial lesion are resolved and cease after a given period of time [44-48], as no further tissue repair can take place. Further linear studies with a more extensive cohorts would be required to ascertain it.

Clinically, however, all of the subjects showed clinical improvement of spasticity post injection of botulin toxin [39].

It is accepted that fractional anisotropy values change with age [49-52] and may also vary according to which of the two main measurement procedures is used. One measurement method is based on tracing regions of interest (ROI) in selected areas, as performed to obtain the white matter tracts and their FA in this pilot study. The other method is based on statistical parametric mapping (SPM), which is a more standardized and automated procedure [53]. In our group of subjects, the youngest showed the highest FA values and no significant post injection changes, which may have been influenced by the time of evolution as

\section{Time vs FA of aff CST pre and post botox}

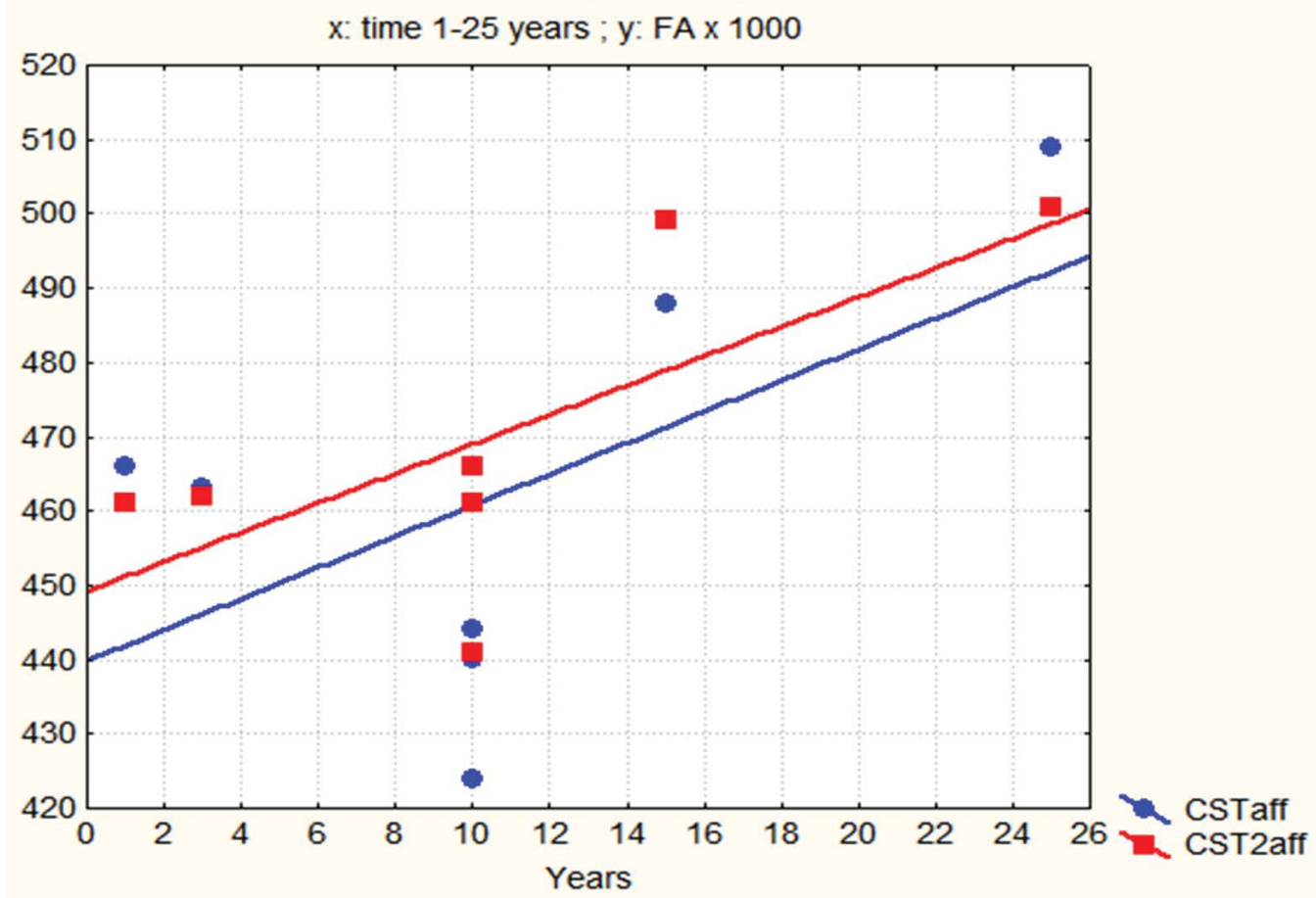

Figure 7: Evolution time range 1-25 years on $\mathrm{x}$-axis vs. fractional anisotropy values of the affected corticospinal tracts pre and post injection of botulin on $y$-axis in all 7 subjects. FA values are multiplied by 1000 to convert them into integers. 
the lesion occurred in early infancy and more than 20 years had elapsed to the development of the episode of handicapping spasticity considered in this study.

FA values in the subjects of this cohort have decreased with age, as noted then plotting age on $x$-axis and pre-post injection FA on y-axis (Figure 7).
Additionally, it was unclear why those subjects with imaging signs of Wallerian degeneration in the affected corticospinal tract showed both clinical and FA post injection improvement as it has been found by other authors [54], although there is contradictory evidence in this regard in the literature $[55,56]$.

\section{Time vs FA of affected CST pre and post botox}

$x$ : time 5-15 years; y: FAx1000

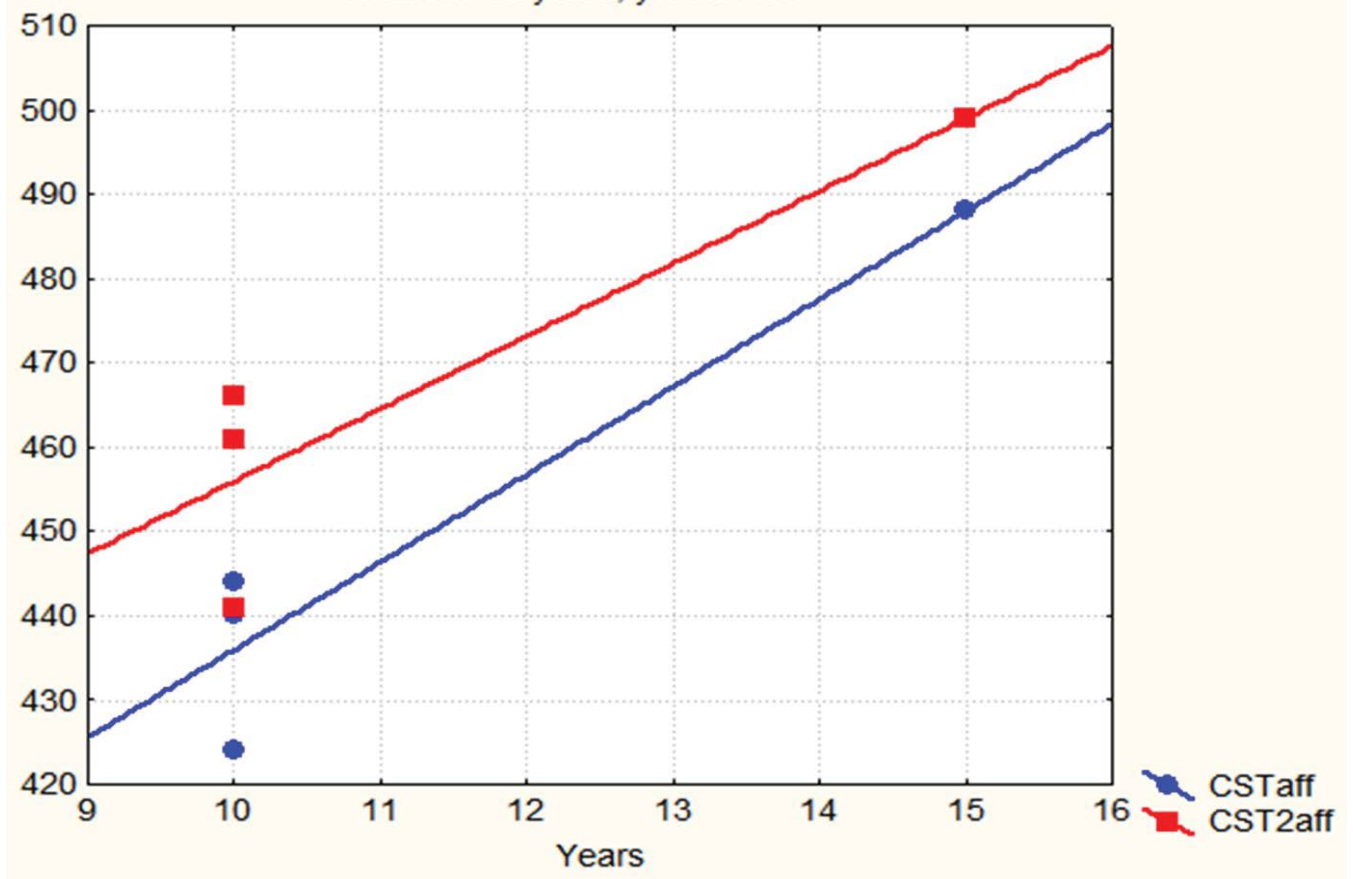

Figure 8: Evolution time range 5-15 years vs. fractional anisotropy values of the affected corticospinal tracts on y-axis, pre and post injection of botulin, in 4 subjects. FA values are multiplied by 1000 to convert them into integers.

Square of FA differences in the affected CST

Double $Y$ plot: age on the left, years of evolution on the right

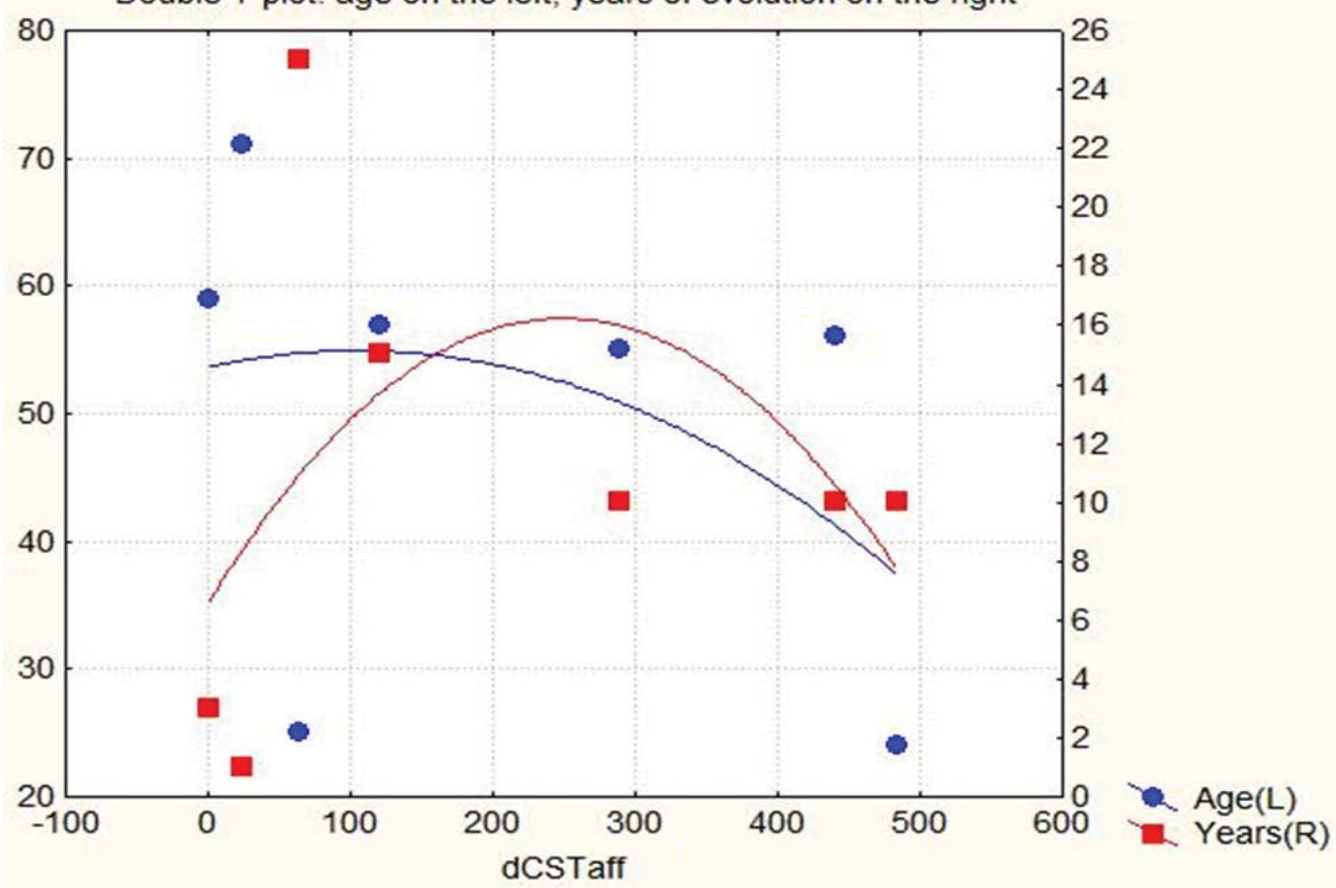

Figure 9: Double $y$-axis plot of square of the FA differences of the affected CST on $x$-axis, versus subjects age, on left $y$-axis, and years of evolution after CVA on right y-axis. (FA values are converted into an integer by multiplying them by 1000 ). 
Square of FA differences in the contralateral CST

Double $Y$ plot: age on the left, years of evolution on the right

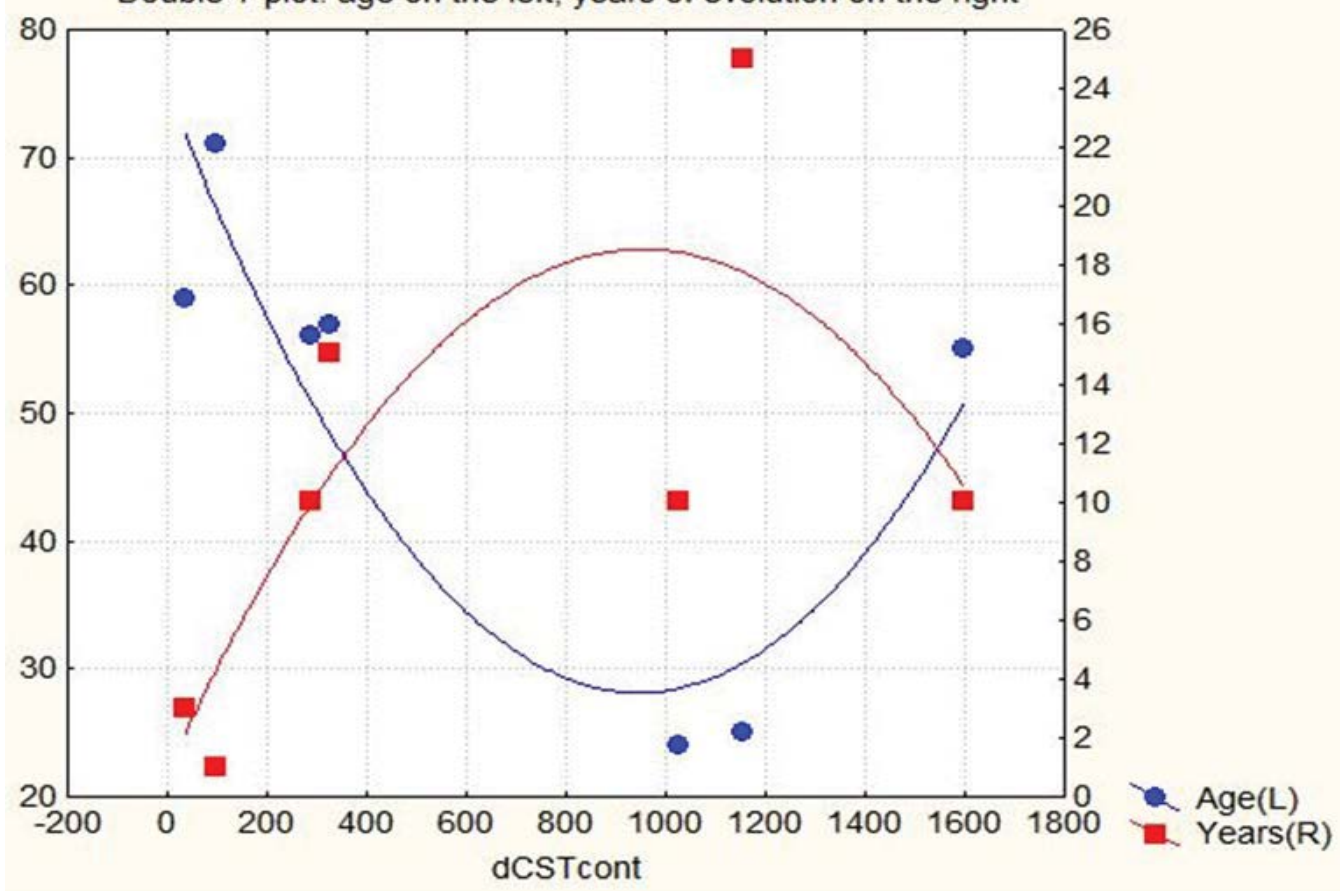

Figure 10: Double $y$-axis plot of square of the FA differences of the contralateral CST on $x$-axis, versus subjects age, on left $y$-axis, and years of evolution after CVA on right $y$-axis. (FA values are converted into an integer by multiplying them by 1000 ).

Direct effects of botulin neurotoxin have been described by some authors [57] suggesting that indirect plasticity of central circuits subsequent to a reduction of sensory input from the injected muscles may be one of the explanations [58-60]. An alternative hypothesis is direct influence of the neurotoxin on central circuits, similar to that seen with tetanus neurotoxin $[61,62]$.

The results of the forward stepwise multiple regression that considered years of evolution as dependent variable and the square of the pre and post injections FA differences for each area, could be due to fractional anisotropy variations related to time elapsed after the lesion, neuroplasticity and influenced by the subjects age (Figure 9 and Figure 10), as the regression did not show significant post injection FA values on the affected side but high correlation $(r)$ and good prediction $\left(r^{2}\right)$ values for structures on the contralateral side.

\section{Conclusion}

Spasticity that developed in a time range from 2 to 22 years after a CVA improved clinically after the peripheral perineural injections of botulin toxin in all 7 subjects of our cohort.

Additionally, 4 out of 7 subjects with lesions acquired 5 to 15 years prior to the development of spasticity showed statistically significant FA changes in the affected corticospinal tracts after the injections of botulin toxin.

3 subjects with lesions occurred outside that range, i.e. less than 5 and more than 15 years, did not show statistically significant FA variations in the corticospinal tracts of the affected sides.

There was regression between years of evolution as a dependent variable and $F A$ values of the contralateral (non-affected) corticospinal tracts, and white matter of the parietal and frontal lobes of the contralateral side, as independent variables.

Further assessments of a larger cohort of patients should be pursued to confirm these preliminary findings.

\section{Acknowledgements}

The authors wish to express their gratitude to Mark Kerr, Librarian of East Kent Hospitals University NHS Foundation Trust, for his assistance with searches and retrievals of references used throughout this research, and also to both $\mathrm{Dr}$ Thomas Webb, Consultant in Stroke Medicine at East Kent Hospitals University NHS Foundation Trust, and Dr. Sofia Bertoni, for their assistance in preparing this manuscript.

\section{References}

1. Ferido T, Habel M (1988) Spasticity in head trauma and CVA patients: Etiology and management. J Neurosci Nurs 20: 17-22.

2. Bertoni MA, Sakel M, Hojjattoleslami A, Bertoni IV, Ratnarajah N (2012) Neuroimaging assessment of spasticity developed after acquired brain injuries and multiple sclerosis. Neuroradiol J 25: 311-317.

3. Agosta F, Pagani E, Petrolini M, Caputo D, Perini M, et al. (2010) Assessment of white matter tract damage in patients with amyotrophic lateral sclerosis: A diffusion tensor MR imaging tractography study. AJNR Am J Neuroradiol 31: $1457-1461$. 
4. Chen F, Zhang X, Li M, Wang R, Wang HT, et al. (2012) Axial diffusivity and tensor shape as early markers to assess cerebral white matter damage caused by brain tumors using quantitative diffusion tensor tractography. CNS Neurosci Ther 18: 667-673.

5. Galantucci S, Tartaglia MC, Wilson SM, Henry ML, Filippi $\mathrm{M}$, et al. (2011) White matter damage in primary progressive aphasias: A diffusion tensor tractography study. Brain 134: 3011-3029.

6. Matsuo K, Mizuno T, Yamada K, Akazawa K, Kasai T, et al. (2008) Cerebral white matter damage in frontotemporal dementia assessed by diffusion tensor tractography. Neuroradiology 50: 605-611.

7. Sarro L, Agosta F, Canu E, Riva N, Prelle A, et al. (2011) Cognitive functions and white matter tract damage in amyotrophic lateral sclerosis: A diffusion tensor tractography study. AJNR Am J Neuroradiol 32: 1866-1872.

8. Trivedi R, Bagga D, Bhattacharya D, Kaur P, Kumar P, et al. (2013) White matter damage is associated with memory decline in chronic alcoholics: A quantitative diffusion tensor tractography study. Behav Brain Res 250: 192-198.

9. Lumsden DE, Ashmore J, Ball G, Charles-Edwards, Selway R, et al. (2016) Fractional anisotropy in children with dystonia or spasticity correlates with the selection for DBS or ITB movement disorder surgery. Neuroradiology 58: 401-408.

10. Alba-Ferrara LM, de Erausquin GA (2013) What does anisotropy measure? Insights from increased and decreased anisotropy in selective fiber tracts in schizophrenia. Front Integr Neurosci 7: 9.

11. El-Serougy L, Abdel Razek AA, Ezzat A, Eldawoody $H$, El-Morsy A (2016) Assessment of diffusion tensor imaging metrics in differentiating low-grade from high-grade gliomas. Neuroradiol J 29: 400-407.

12. Razek A, El-Serougy L, Abdelsalam M, Gaballa G, Talaat M (2018) Differentiation of residual/recurrent gliomas from postradiation necrosis with arterial spin labeling and diffusion tensor magnetic resonance imaging-derived metrics. Neuroradiology 60: 169-177.

13. Razek A, Shabana AAE, El Saied TO, Alrefey N (2017) Diffusion tensor imaging of mild-moderate carpal tunnel syndrome: Correlation with nerve conduction study and clinical tests. Clin Rheumatol 36: 2319-2324.

14. Hecke A, Weiler M, Xia A, Ruetters M, Pham M, et al. (2015) Peripheral nerve diffusion tensor imaging: Assessment of axon and myelin sheath integrity. PLOS One.

15. Sijens PE, Mostert JP, Irwan R, Potze JH, Oudkerk M, et al. (2008) Impact of fluoxetine on the human brain in multiple sclerosis as quantified by proton magnetic resonance spectroscopy and diffusion tensor imaging. Psychiatry Res 164: 274-282.

16. Teipel SJ, Grothe MJ, Filippi M, Fellgiebel A, Dyrba M, et al. (2014) Fractional anisotropy changes in Alzheimer's disease depend on the underlying fiber tract architecture: A multiparametric DTI study using joint independent component analysis. J Alzheimers Dis 41: 69-83.

17. Harris NG, Verley DR, Gutman BA, Sutton RL (2016) Bidirectional changes in fractional anisotropy after experiment TBI: Disorganization and reorganization? Neuroimage 33: 129-143.

18. Horsfield MA, Lai M, Webb SL, Barker GJ, Tofts PS, et al. (1996) Apparent diffusion coefficients in benign and secondary progressive multiple sclerosis by nuclear magnetic resonance. Magn Reson Med 36: 393-400.
19. Wheeler-Kingshott CA, Parker GJ, Symms MR, Hickman SJ, Tofts PS, et al. (2002) ADC mapping of the human optic nerve: Increased resolution, coverage, and reliability with CSF-suppressed ZOOM-EPI. Magn Reson Med 47: 24-31.

20. Brashear A, Zafonte R, Corcoran M, Galvez-Jimenez N, Gracies JM, et al. (2002) Inter- and intrarater reliability of the ashworth scale and the disability assessment scale in patients with upper-limb poststroke spasticity. Arch Phys Med Rehabil 83: 1349-1354.

21. Ratnarajah N, Simmons A, Bertoni M, Hojjatoleslami A (2013) Two-tensor model-based bootstrapping on classified tensor morphologies: Estimation of uncertainty in fiber orientation and probabilistic tractography. Magn Reson Imaging 31: 296-312.

22. Baba Y, Osborne M, Wszolek Z, Kwolek A, Druzbicki M (2004) Treatment of spasticity with botulinum toxin. Ortop Traumatol Rehabil 6: 665-672.

23. Leathley MJ, Gregson JM, Moore AP, Smith TL, Sharma AK, et al. (2004) Predicting spasticity after stroke in those surviving to 12 months. Clin Rehabil 18: 438-443.

24. Clemenzi A, Formisano R, Matteis M, Gallinacci L, Cochi G, et al. (2012) Care management of spasticity with botulinum toxin-a in patients with severe acquired brain injury: A 1-year follow-up prospective study. Brain Inj 26: 979-983.

25. D'Epiro S, Macaluso L, Salvi M, Luci C, Mattozzi C, et al. (2014) Safety and prolonged efficacy of botulin toxin a in primary hyperhidrosis. Clin Ter 165: e395-e400.

26. Grandas F, Elston J, Quinn N, Marsden CD (1989) Pharmacologic, surgical and infiltration of botulin toxin treatment in blepharospasm. Neurologia 4: 194-199.

27. Milinis K, Tennant A, Mills RJ, Al-Chalabi A, Burke G, et al. (2018) Development and validation of spasticity indexamyotrophic lateral sclerosis. Acta Neurol Scand 138: 47-54.

28. Fox RJ, Sakaie K, Lee JC, Debbins JP, Liu Y, et al. (2012) A validation study of multicenter diffusion tensor imaging: Reliability of fractional anisotropy and diffusivity values. AJNR Am J Neuroradiol 33: 695-700.

29. Soriano-Raya JJ, Miralbell J, López-Cancio E, Bargalló N, Arenillas JF, et al. (2014) Tract-specific fractional anisotropy predicts cognitive outcome in a community sample of middle-aged participants with white matter lesions. J Cereb Blood Flow Metab 34: 861-869.

30. Chang CL, Weber DJ, Munin MC (2015) Changes in cerebellar activation after onabotulinumtoxina injections for spasticity after chronic stroke: A pilot functional magnetic resonance imaging study. Arch Phys Med Rehabil 96: 2007-2016.

31. Kikuchi Al, Takeda A, Sugeno N, Miura E, Kato K, et al. (2016) Brain metabolic changes of cervical dystonia with spinocerebellar ataxia type 1 after botulinum toxin therapy. Intern Med 55: 1919-1922.

32. Cocco A, Albanese A (2018) Recent developments in clinical trials of botulinum neurotoxins. Toxicon 147: 77-83.

33. Hallett M (2018) Mechanism of action of botulinum neurotoxin: Unexpected consequences. Toxicon 147: 73-76.

34. Kanovsky P, Rosales RL (2011) Debunking the pathophysiological puzzle of dystonia-with special reference to botulinum toxin therapy. Parkinsonism Relat Disord 1: S11-S14.

35. Saita K, Morishita T, Hyakutake K, Fukuda H, Shiota E, et al. (2017) Combined therapy using botulinum toxin $A$ and single-joint hybrid assistive limb for upper-limb disability due to spastic hemiplegia. J Neurol Sci 373: 182-187. 
36. Senkarova Z, Hlustik P, Otruba P, Herzig R, Kanovsky P (2010) Modulation of cortical activity in patients suffering from upper arm spasticity following stroke and treated with botulinum toxin a: An fmri study. J Neuroimaging 20: 9-15.

37. Manganotti $P$, Acler M, Formaggio E, Avesani M, Milanese $F$, et al. (2010) Changes in cerebral activity after decreased upper-limb hypertonus: An EMG-fmri study. Magn Reson Imaging 28: 646-652.

38. Di Stadio A, Bernitsas E, Restivo DA, Alfonsi E, MarcheseRagona $\mathrm{R}$ (2018) Spasmodic dysphonia in multiple sclerosis treatment with botulin toxin a: A pilot study. J Voice.

39. Kwiecien-Czerwieniec I, Krukowska J, Woldanska-Okonska M (2014) Influence of toxin botulin on walk stereotype of children with juvenile cerebral palsy. The functional examination performed by BTS, comprehensive movement analysis system. A preliminary report. Wiad Lek 67: 59-63.

40. Struys MA, Devriese PP, Ongerboer de Visser BW, Speelman JD, de Jong PT (1991) The treatment of hemifacial spasms using botulin. Ned Tijdschr Geneeskd 135: 889-892.

41. Aguilar-Rebolledo F, Hernández-Sánchez J, Rayo-Mares D, Soriano-Fonseca F, García-Muñoz L, et al. (2001) Botulin toxin as treatment for spasticity and dystonia in infantile cerebral paralysis. Gac Med Mex 137: 403-411.

42. Surova Y, Nilsson M, Lampinen B, Lätt J, Hall S, et al. (2018) Alteration of putaminal fractional anisotropy in Parkinson's disease: A longitudinal diffusion kurtosis imaging study. Neuroradiology 60: 247-254.

43. Drobyshevsky A (2017) Concurrent decrease of brain white matter tract's thicknesses and fractional anisotropy after antenatal hypoxia-ischemia detected with tract-based spatial statistics analysis. J Magn Reson Imaging 45: 829-838.

44. Bowman EA, Velakoulis $D$, Desmond $P$, Walterfang $M$ (2018) Longitudinal changes in white matter fractional anisotropy in adult-onset niemann-pick disease type c patients treated with miglustat. JIMD Rep 39: 39-43.

45. Garcia-Lazaro HG, Becerra-Laparra I, Cortez-Conradis D, Roldan-Valadez E (2016) Global fractional anisotropy and mean diffusivity together with segmented brain volumes assemble a predictive discriminant model for young and elderly healthy brains: A pilot study at $3 \mathrm{~T}$. Funct Neurol 31: 39-46.

46. Moore E, Schaefer RS, Bastin ME, Roberts N, Overy K (2017) Diffusion tensor MRI tractography reveals increased fractional anisotropy (FA) in arcuate fasciculus following music-cued motor training. Brain Cogn 116: 40-46.

47. Van Impe A, Coxon JP, Goble DJ, Doumas M, Swinnen SP (2012) White matter fractional anisotropy predicts balance performance in older adults. Neurobiol Aging 33: 1900-1912.

48. Yu C, Shu N, Li J, Qin W, Jiang T, et al. (2007) Plasticity of the corticospinal tract in early blindness revealed by quantitative analysis of fractional anisotropy based on diffusion tensor tractography. Neuroimage 36: 411-417.
49. Bisdas S, Bohning DE, Besenski N, Nicholas JS, Rumboldt Z (2008) Reproducibility, interrater agreement, and agerelated changes of fractional anisotropy measures at $3 T$ in healthy subjects: Effect of the applied b-value. AJNR Am J Neuroradiol 29: 1128-1133.

50. Grieve SM, Williams LM, Paul RH, Clark CR, Gordon E (2007) Cognitive aging, executive function, and fractional anisotropy: A diffusion tensor MR imaging study. AJNR Am J Neuroradiol 28: 226-235.

51. Mamata H, Jolesz FA, Maier SE (2005) Apparent diffusion coefficient and fractional anisotropy in spinal cord: Age and cervical spondylosis-related changes. J Magn Reson Imaging 22: 38-43.

52. Schneider J, Kober T, Bickle Graz M, Meuli R, Hüppi PS, et al. (2016) Evolution of T1 relaxation, ADC, and fractional anisotropy during early brain maturation: A serial imaging study on preterm infants. AJNR Am J Neuroradiol 37: 155162.

53. Furutani $K$, Harada $M$, Minato $M$, Morita $N$, Nishitani $H$ (2005) Regional changes of fractional anisotropy with normal aging using statistical parametric mapping (SPM). J Med Invest 52: 186-190.

54. Sawlani V, Gupta RK, Singh MK, Kohli A (1997) MRI demonstration of wallerian degeneration in various intracranial lesions and its clinical implications. J Neurol Sci 146: 103-108.

55. Kleinman JT, Gailloud P, Jordan LC (2010) Recovery from spatial neglect and hemiplegia in a child despite a large anterior circulation stroke and wallerian degeneration. $J$ Child Neurol 25: 500-503.

56. Miyai I, Suzuki T, Mikami A, Kubota K, Volpe BT (2001) Patients with capsular infarct and wallerian degeneration show persistent regional premotor cortex activation on functional magnetic resonance imaging. J Stroke Cerebrovasc Dis 10: 210-216.

57. Caleo M, Restani L (2018) Direct central nervous system effects of botulinum neurotoxin. Toxicon 147: 68-72.

58. Curra A, Trompetto C, Abbruzzese G, Berardelli A (2004) Central effects of botulinum toxin type a: Evidence and supposition. Mov Disord 8: S60-S64.

59. Filippi GM, Errico P, Santarelli R, Bagolini B, Manni E (1993) Botulinum a toxin effects on rat jaw muscle spindles. Acta Otolaryngol 113: 400-404.

60. Rosales RL, Arimura K, Takenaga S, Osame M (1996) Extrafusal and intrafusal muscle effects in experimental botulinum toxin-a injection. Muscle Nerve 19: 488-496.

61. Laura Restani, Francesco Giribaldi, Maria Manich, Kinga Bercsenyi, Guillermo Menendez, et al. (2012) Botulinum neurotoxins a and e undergo retrograde axonal transport in primary motor neurons. PLOS Pathog 8.

62. Bomba-Warczak E, Vevea JD, Brittain JM, FigueroaBernier A, Tepp WH, et al. (2016) Interneuronal transfer and distal action of tetanus toxin and botulinum neurotoxins $a$ and d in central neurons. Cell Rep 16: 1974-1987. 